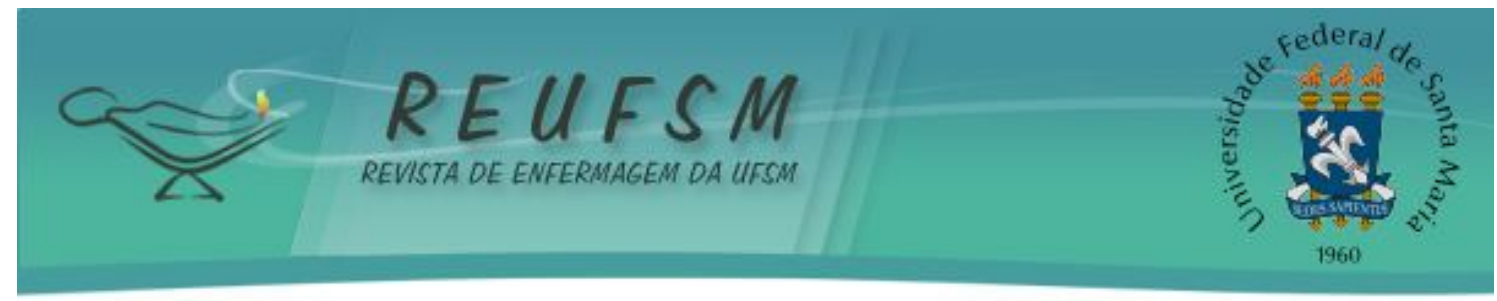

ARTIGO ORIGINAL

\title{
PERCEPÇÃO DE PACIENTES COM NEOPLASIAS ESOFÁGICAS E ESTOMACAIS SOBRE ORIENTAÇÕES PRÉ-OPERATÓRIAS RECEBIDAS DO ENFERMEIRO
}

\author{
PATIENTS WITH ESOPHAGEAL AND ST OMACH NEOPLASMS PERCEPTIONS ABOUT \\ GUIDELINES ON PREOPERATIVE RECEIVED FROM
}

\section{PERCEPCIÓN DE LOS PACIENTES CON NE OPLASIAS ESOFÁGICAS Y EST OMACALES SOBRE ORIENT ACIONES PREOPERAT ORIAS RECIBIDAS DEL ENFERMERO}

\author{
Rodrigo Marques da Silva ${ }^{1}$ \\ Rhea Silvia de Ávila Soares ${ }^{2}$ \\ Letice Dalla Lana ${ }^{3}$ \\ J ucelaine Arend Birrer ${ }^{4}$ \\ Sadja Cristina Tassinari de Souza Mostardeiro ${ }^{5}$
}

RESUMO: Objetivo: conhecer as percepções dos pacientes com neoplasias gástricas e esofágicas sobre as orientações pré-operatórias para as cirurgias de Gastrectomia e Esofagectomia. Método: Estudo qualitativo e descritivo, cuja coleta de dados foi realizada entre novembro de 2010 a janeiro de 2011 por meio de entrevista estruturada junto a oito pacientes internados na clínica cirúrgica do Hospital Universitário de Santa Maria, Rio Grande do Sul. As informações foram interpretadas mediante análise de conteúdo. Resultados: evidenciaram-se quatro categorias: Sentimentos revelados pelo paciente com relação à orientação recebida; Percepção do paciente frente ao profissional que realizou a orientação; Sentido das orientações em saúde para os pacientes; e Sentimentos vivenciados pelo paciente em relação à alta hospitalar. Conclusões: os pacientes receberam orientações adequadas sobre suas cirurgias e sentiram-se mais tranquilos e seguros em relação ao procedimento cirúrgico.

Descritores: Enfermagem; Cuidados de enfermagem; Cuidados pré-operatórios.

ABSTRACT: Objective: to understand patients with gastric and esophageal neoplasms perceptions about the preoperative guidelines to gastrectomy and esophagectomy surgeries. Method: This is a descriptive qualitative study, which the data was collected between November 2010 and J anuary 2011 through a structured interview was used along with eight patients in the surgical clinic of the University Hospital of Santa Maria, Rio Grande do Sul. The information was interpreted through content analysis. Results: four categories emerged: feelings revealed by the patient in relation to orientation received; patient's perceptions towards the professional who conducted the orientation; Health guidance meaning for patients; and feelings experienced by patient about hospital

\footnotetext{
${ }^{1}$ Acadêmico do 8 o semestre do Curso de Enfermagem da Universidade Federal de Santa Maria. Bolsista PIBIC/ CNPQ. Integrante do Grupo de Pesquisa Trabalho, Saúde, Educação e Enfermagem. Linha de Pesquisa Stress, Coping e Burnout. E-mail: marques-sm@hotmail.com.

2 Enfermeira do Hospital Universitário de Santa Maria (HUSM), Unidade de Clinica Cirúrgica, Especialista em Saúde Pública pela Faculdade Internacional de Curitiba, Coordenadora do Grupo de Estudos de Lesões de Pele (GELP) do HUSM. E-mail: rheasilviasoares@yahoo.com.br.

${ }^{3}$ Enfermeira, Residente do Programa de Residência Multiprofissional Integrada em Sistema Público de Saúde da UFSM. E-mail: leticedl@hotmail.com.

${ }^{4}$ Enfermeira do Hospital Universitário de Santa Maria (HUSM), Unidade de Internação Cirúrgica, Especialista em Gestão da Clínica dos Hospitais do SUS, Preceptora da Residência Multiprofissional Integrada em Sistema Público de Saúde do CCS/ UFSM/ HUSM, Mestranda em Administração pela UFSM. E-mail: juarend@ibest.com.br ${ }^{5}$ Doutora em enfermagem. Professora adjunta do Curso de Enfermagem da Universidade Federal de Santa Maria-RS (UFSM). E-mail: sadjasm@yahoo.com.br.
} 


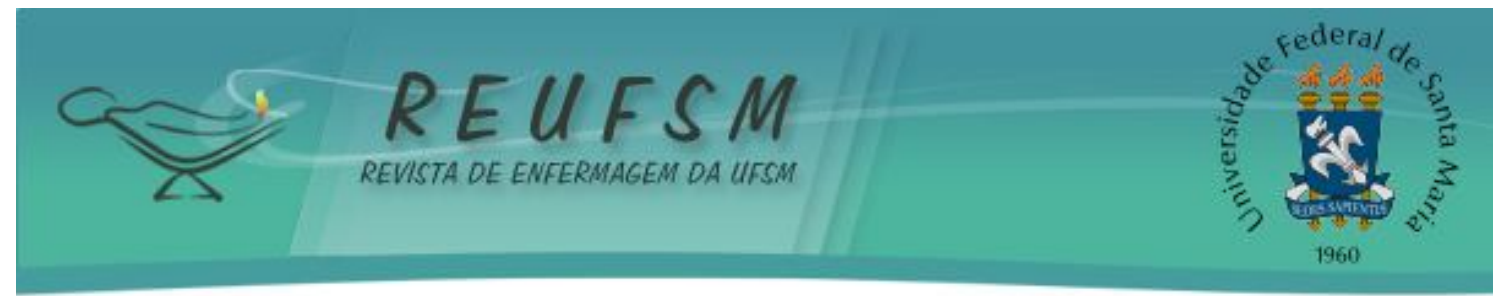

discharge. Conclusions: patients received appropriate guidance about their surgeries and felt more calm and confident in relation the surgical procedure.

Descriptors: Nursing; Nursing care; Preoperative care.

RESUMEN: Objetivo: conocer las percepciones de los pacientes con neoplasias gástricas y esofágicas sobre las orientaciones preoperatorias para las cirugías de gastrectomía y esofagectomía. Método: Estudio cualitativo y descriptivo, cuya recogida de datos fue realizada entre noviembre de 2010 hasta enero de 2011 por medio de entrevista estructurada junto a ocho pacientes internados en la clínica quirúrgica del Hospital Universitario de Santa Maria, Rio Grande del Sur. Las informaciones fueron interpretadas por análisis de contenido. Resultados: se evidenció cuatro categorías: sentimientos que fueron dichos por el paciente sobre la orientación recibida, la percepción del paciente acerca del profesional que realizó la orientación, el sentido de la orientación de salud para los pacientes y los sentimientos experimentados por el paciente en relación a la alta hospitalaria. Conclusión: los pacientes recibieron una orientación adecuada sobre sus cirugías y se sintieron más tranquilos y confiados sobre el procedimiento quirúrgico.

Descriptores: Enfermería; Atención de enfermería; Cuidados preoperatorios.

\section{INTRODUÇÃO}

As concepções de cuidado estão presentes no debate sobre a qualidade da atenção à saúde, campo no qual a Enfermagem se articula para o desenvolvimento de sua prática e tem 0 cuidado como seu objeto de trabalho. ${ }^{1}$ Dentre os cuidados que cabem ao enfermeiro, destaca-se a educação em saúde, conceituada como uma relação permeada por um conjunto de valores, práticas sociais, costumes e tradições que fazem parte da formação cultural de cada sujeito. ${ }^{2}$ Nesse processo educativo, as pessoas se informam e adquirem habilidades para fazer escolhas saudáveis sobre sua vida, o que contribui para a ocorrência de mudanças que favoreçam a melhoria da saúde. ${ }^{3}$

Nesse contexto, as práticas educativas em saúde são uma realidade efetiva, principalmente, devido às mudanças dos paradigmas de atenção à saúde e a implantação do conceito da promoção da saúde. ${ }^{4} \mathrm{~A}$ abordagem do enfermeiro deve ser participativa, evitar a redução à assistência curativa, desenvolver ações de promoção à saúde e garantir um modelo assistencial integrado para responder às necessidades individuais e coletivas. ${ }^{5}$ Assim, a educação em saúde representa um instrumento facilitador para a capacitação da comunidade e contribui para a promoção da saúde. ${ }^{5}$

Dessa maneira, esse profissional de saúde e os pacientes precisam estabelecer uma relação dialógica pautada na escuta terapêutica, no respeito e na valorização das experiências de cada paciente. Para desenvolver estas ações, é necessário que o enfermeiro conheça as práticas educativas e interaja com os pacientes para reconstrução coletiva de saberes e práticas. ${ }^{5}$

0 processo educativo pode e deve ser desempenhado em todos os momentos e espaços. ${ }^{6}$ Dentre eles, destaca-se a Unidade de Clínica Cirúrgica, local em que o ensinoaprendizagem do paciente internado começa no pré-operatório. Nesse período, o enfermeiro deve estabelecer vínculo com o paciente e a família para ajudá-los a compreender a sua situação clínica, o que se dá por meio das orientações pré-operatórias. Tal ação tem por finalidade a adaptação do paciente à mudança do estilo de vida, que surgirá após a cirurgia, para que, no momento da alta hospitalar, ele e seu cuidador estejam preparados para os cuidados no domicílio. ${ }^{7}$ 


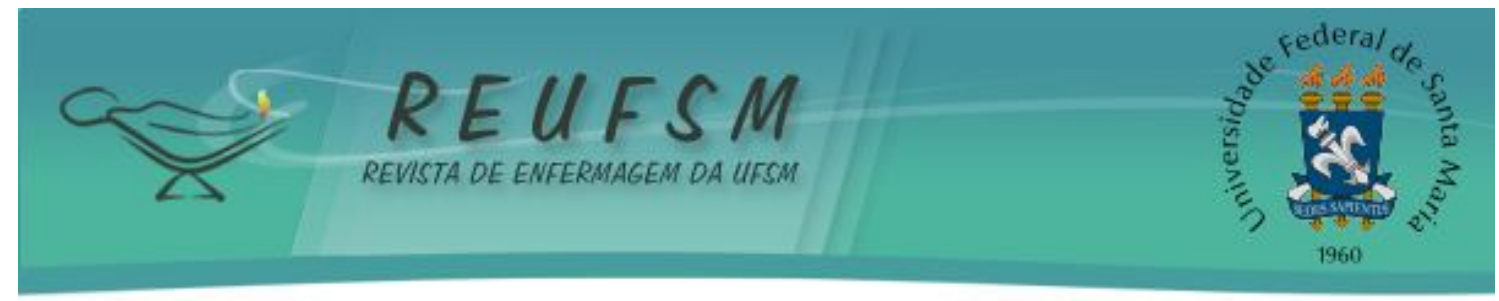

A intervenção cirúrgica é, em geral, uma experiência emocionalmente difícil para o ser humano. Os indivíduos podem apresentar um estado de tensão, ansiedade ou medo em decorrência do desconhecido, da anestesia, da mutilação e até mesmo da possibilidade de morte. ${ }^{8}$ Por isso, o enfermeiro é o profissional responsável pelo planejamento da assistência de enfermagem e tomada de decisão sobre o cuidado prestado ao paciente cirúrgico. ${ }^{9}$

As orientações são realizadas pelos enfermeiros das Unidades de Clínicas Cirúrgicas, onde se prestam cuidados no pré-operatório mediato e imediato. 0 primeiro compreende desde o momento da indicação cirúrgica até 24 horas antes do ato cirúrgico. 0 último envolve desse momento até o encaminhamento do paciente ao centro cirúrgico. ${ }^{7}$

As orientações pré-operatórias auxiliam o paciente a lidar com a cirurgia, reduzem a duração da internação hospitalar, elevam a satisfação com o serviço prestado, minimizam complicações cirúrgicas e aumentam o bem estar psíquico do paciente. ${ }^{4}$ Para atingir esses objetivos, tais orientações precisam ser individualizadas, atender as necessidades de cada paciente, considerar o conhecimento prévio e responder às dúvidas do mesmo. No entanto, durante a execução dessa atividade, são fornecidas informações que, por vezes, não são assimiladas pelo paciente e, por isso, os questionamentos sobre o ato cirúrgico podem não ser feitos por ele. Além disso, para alguns autores, as complicações no pós-operatório estão, geralmente, associadas a um preparo inadequado antes do ato cirúrgico. ${ }^{7}$ Logo, esse profissional precisa instrumentalizar-se durante a graduação e em serviço para tal. ${ }^{6}$

No hospital estudado, são realizados processos cirúrgicos de várias especialidades. Contudo, para essa pesquisa, utilizaram-se os pacientes submetidos à esofagectomia e gastrectomia, pois ambas as cirurgias são realizadas com frequência nessa instituição. ${ }^{10}$

No ano de 2009, foram realizadas 27 cirurgias de esofagectomia e 10 de gastrectomia, ou seja, uma média de 2 cirurgias de esofagectomia por mês e 1 por mês nas cirurgias de gastrectomia, conforme setor de estatística do referido hospital. Tais informações demonstram a necessidade de um olhar mais atento para estes pacientes.

Conforme dados do setor estatístico do hospital ${ }^{10}$, esses procedimentos cirúrgicos são realizados, com frequência, em pacientes com neoplasias gástricas ou esofágicas. Além disso, sabe-se que a estimativa de novos casos de câncer de esôfago é de 18,5 para cada 100.000 pessoas e de câncer de estômago de 16,58 para cada 100.000 no Rio Grande do Sul. ${ }^{11}$

A partir do exposto, busca-se conhecer as percepções dos pacientes, com diagnóstico de neoplasia de esôfago ou estômago, sobre as orientações pré- operatórias recebidas de enfermeiros para as cirurgias de Gastrectomia e Esofagectomia em um Hospital Universitário do Interior do Rio Grande do Sul.

Ao avaliar as orientações do ponto de vista dos pacientes, espera-se saber se eles têm compreendido as informações que recebem e se elas têm auxiliado no pós-operatório.

Além disso, deseja-se verificar se as estratégias e/ ou metodologias utilizadas pelos enfermeiros nas orientações pré-operatórias atendem as necessidades de cada paciente e se atingem os obj etivos propostos por essa atividade.

\section{MÉTODO}

Trata-se de uma pesquisa descritiva, com abordagem qualitativa, realizada entre Novembro de 2010 a Janeiro de 2011. O cenário do estudo foi o Hospital Universitário de Santa Maria (HUSM), onde se desenvolve ensino, pesquisa e extensão por meio da assistência à comunidade na área da saúde. Este hospital localiza-se no interior do Estado do Rio Grande do Sul (RS), é considerado referência de média e alta complexidade para região centro-oeste do estado e abrange 46 municípios. 


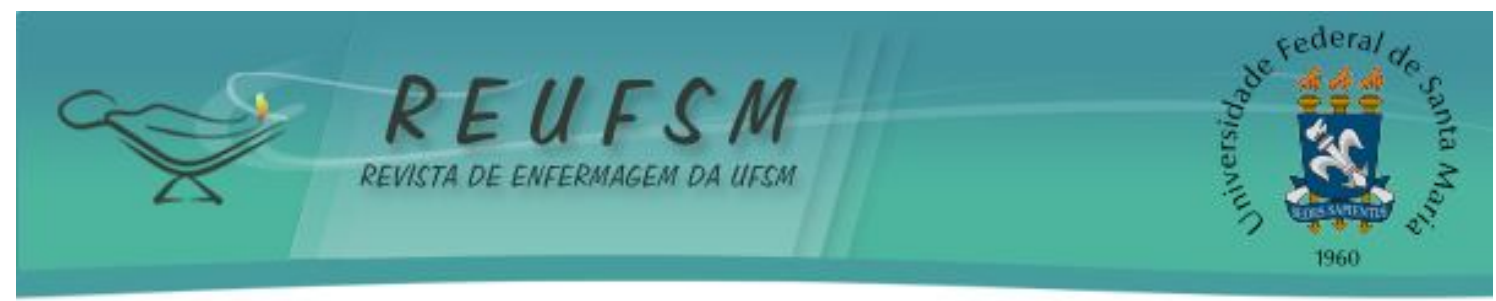

O convite aos pacientes foi realizado por meio de visita à unidade de internação. Nesse momento, informaram-se os obj etivos da investigação e a forma de participação.

Inicialmente, os enfermeiros da unidade realizaram as orientações pré-operatórias aos pacientes com indicação para as cirurgias de esofagectomia e gastrectomia. No pósoperatório dos mesmos, procedeu-se a coleta de dados, que ocorreu por meio de entrevista estruturada com quatro questões abertas. 0 registro das entrevistas foi feito com uso de um gravador após autorização do paciente. Além disso, entregaram-se aos entrevistados um questionário para levantamento de dados gerais.

Para evitar constrangimentos e a indução de respostas, o questionário foi aplicado por outro enfermeiro, que não o que realizou as orientações.

Incluíram-se no estudo, pacientes maiores de 18 anos, que aceitaram participar da pesquisa, internados na Unidade de Clínica Cirúrgica do referido hospital no período da coleta de dados, com diagnóstico médico de neoplasia esofágica ou gástrica e indicação de esofagectomia e gastrectomia, respectivamente. Excluíram-se da pesquisa, pacientes que apresentaram alguma complicação pós-operatória e não puderam participar da entrevista.

Após a coleta dos dados, as entrevistas foram transcritas e analisadas segundo a Análise de Conteúdo proposta por Bardin. ${ }^{12}$ Utilizou-se essa técnica, pois os procedimentos envolvidos são estruturados de forma a promover a organização dos dados através de fases ou etapas que levam a um resultado estrutural mente organizado do seu conteúdo. ${ }^{12}$

Foi assegurado, ainda, o anonimato dos participantes, os quais foram codificados pela letra inicial $F$, referente à palavra "FALA", seguida de um algarismo numérico para diferenciá-los entre si, o qual se referia ao número da entrevista respondida pelo paciente.

Em observância às Diretrizes da Resolução 196/96 $6^{(13)}$ do Conselho Nacional de Saúde, o projeto foi apresentado ao Comitê de Ética em Pesquisa da Universidade Federal de Santa Maria (UFSM) e aprovado em 08 de novembro de 2010 (CAAE: 0205. 0.243.000-10). Utilizou-se o Termo de Consentimento Livre e Esclarecido(TCLE) para os pacientes que concordaram, livremente, em participar da pesquisa.

Selecionaram-se dez pacientes com as cirurgias de gastrectomia ou esofagectomia agendadas no período do estudo. Desses, um foi a óbito e outro necessitou de cuidados intensivos no pós-operatório imediato e tardio (uso de tubo orotraqueal e ventilação mecânica). Consequentemente, esses foram excluídos conforme os critérios selecionados. Assim, entrevistaram-se oito pacientes no pós-operatório dessas cirurgias.

\section{RESULTADOS E DISCUSSÃO}

Dos oito participantes da pesquisa, seis eram do sexo masculino, três possuíam Ensino Fundamental Incompleto, cinco eram casados e três aposentados. A faixa etária ficou entre 41 e 78 anos. Após análise, emergiram quatro categorias temáticas: Sentimentos revelados pelo paciente com relação à orientação recebida; Percepção do paciente frente ao profissional que realizou as orientações pré- operatórias; Sentido das orientações em saúde para os pacientes (subcategorias Sentido das orientações antes da cirurgia e sentido das orientações após a cirurgia); e Sentimentos vivenciados pelo paciente em relação à alta hospitalar.

Para a 1a Categoria, Sentimentos revelados pelo paciente com relação à orientação recebida, os sentimentos observados foram a Segurança e a Tranquilidade.

A prática em saúde possui uma dimensão cuidadora que produz relações intercessoras com o mundo subjetivo dos pacientes, posicionamento ético e articulação dos saberes que compõe os projetos terapêuticos. ${ }^{14} \mathrm{~A}$ orientação pré-operatória exige um diálogo com fala e escuta, em que o paciente reconhece o profissional como referência e sente-se mais tranquilo para a realização do procedimento indicado. Além disso, o 


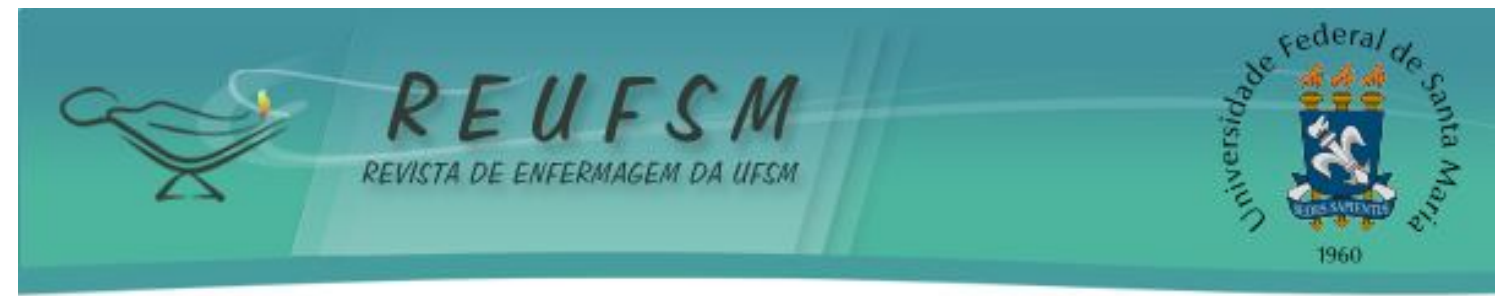

fornecimento de esclarecimentos e orientações sobre a necessidade do procedimento, conforme estudos, tranquilizam o paciente. ${ }^{15}$ Tal observação foi constatada na pesquisa pela fala:

me senti mais seguro mais tranqüilo (F1)

Percebeu-se também que o paciente sente-se mais seguro quando informado sobre os aspectos e procedimentos que envolvem o ato cirúrgico. Isso é exposto na seguinte fala:

[...] Fiquei mais segura, pois sabia o que ia acontecer comigo [...](F2)

Tal sentimento foi encontrado em outro estudo ${ }^{16}$ relacionado à orientação préoperatória. Neste trabalho, o autor destaca que a preparação psicológica é vista como benéfica se baseada nas necessidades individuais do paciente, pois, ao sentir-se esclarecido de suas dúvidas, diminuem-lhe os temores, 0 que previne possíveis complicações no pós-operatório.

Para obtenção desses resultados, é preciso de uma interação profissionalpaciente de qualidade, o que confirma que o trabalho em saúde possui dimensão social e humana. ${ }^{17}$ Portanto, quando se estabelece o cuidado, não se deve priorizar a ação técnica, mas perceber a subjetividade do outro, ol har e ouvir com atenção. ${ }^{17}$

Sobre a segunda categoria, Percepção do paciente frente ao profissional que realizou as orientações pré-operatórias, observaram-se a atenção, a simpatia e a bondade como características atribuídas ao profissional pelos pesquisados quando questionados sobre a relação com o profissional que executou as orientações.

[...] ela foi muito simpática e atenciosa. (F5)

[...] Sinto-me muito agradecida. (F6)

[...] foi muito boa e atenciosa. São maravilhosas e explicam tudo [... ](F7)

O enfermeiro, ao realizar a orientação pré-operatória, deve ser cordial e mostrar-se disponível ao paciente, encorajar a verbalização e ouvir atentamente suas dúvidas e preocupações. A prática do ensino pré-operatório, além de contribuir para a recuperação do paciente, estreita seus vínculos com o enfermeiro e prioriza o homem e não sua enfermidade. ${ }^{18}$

Em relação à 3a categoria, Sentidos das orientações para os pacientes, após análise, emergiram duas subcategorias: Sentido das orientações antes da cirurgia e sentido das orientações após a cirurgia.

Para a primeira subcategoria, Sentido das orientações antes da cirurgia, observou-se que o sentido principal das orientações refere-se às informações que os pacientes buscam e ao conhecimento a que se propõe essa atividade. Isso pode ser observado pelas falas obtidas quando questionados sobre a importância das orientações antes da cirurgia:

Sim, me deixa mais tranquilo sabendo o que ia acontecer. (F1)

Sim, porque fiquei por dentro do que ia fazer. (F2)

Sim, ajuda saber o que vai acontecer. (F6)

Sim, porque agente esta sabendo o que é feito. (F7)

Além disso, pode-se inferir que os profissionais de saúde utilizaram uma linguagem clara e que atendeu as dúvidas e anseios levantados pelos pacientes. Tal fato é identificado no seguinte relato: "Sim, porque me deixou mais a parte da minha situação" 


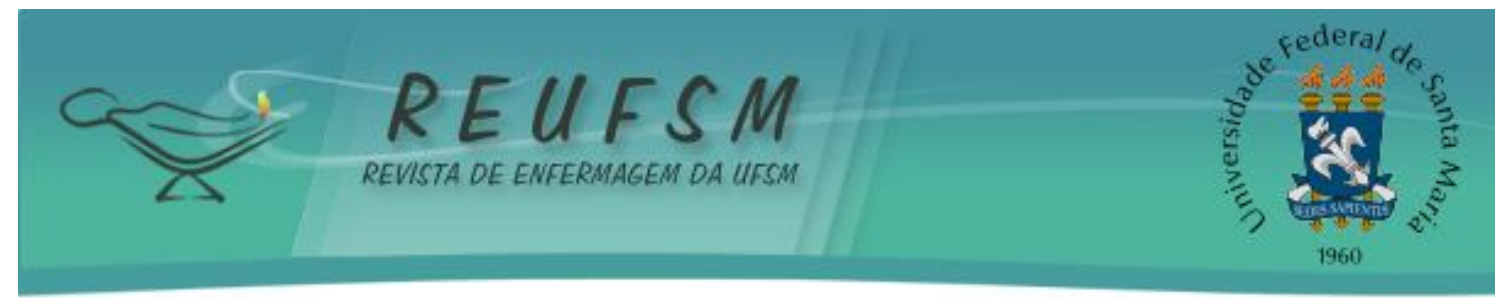

(F2). Sobre isso, estudos apontam que as orientações devem ocorrer no pré-operatório mediato para que o paciente assimile as informações e tenha tempo de esclarecer suas dúvidas. Não obstante, é importante observar o emprego de termos acessíveis aos pacientes, evitar uma linguagem técnico-científica que dificulte o entendimento e que cause angústia e medo. ${ }^{15,17}$

Ainda sobre isso, uma pesquisa ${ }^{15}$ com pacientes de um hospital de ensino internados em uma clínica cirúrgica evidenciou uso de linguagem inadequada no discurso de uma paciente, que relatou haver discordâncias entre as informações fornecidas pelos profissionais, bem como dificuldade de compreendê-las.

Embora se tenha identificado que as orientações explicaram o procedimento cirúrgico com uso de linguagem clara, não se sabe se houve efetiva troca de saberes. A fala abaixo pressupõe que a informação recebida é aquela considerada necessária para o enfermeiro.

\section{[... ]São ótimas, falam tudo, porque elas sabem o que é melhor pra nós (F6)}

Talvez o fato de não haver preparo ou treinamento específico para realização de orientações pré- operatórias influencie no modo como os profissionais realizam essa atividade. Isso pode dificultar a execução de uma orientação adequada às necessidades de cada paciente. Além disso, tradicionalmente, a orientação pré-operatória é feita de modo semelhante a todos, independente do paciente ou da cirurgia a que será submetido, com informações previamente determinadas como importantes pelo profissional. Isso leva à falta de individualização das orientações.

Assim, o modo como o paciente é orientado, muitas vezes, faz com que ele se torne agente passivo do ensino, não se sinta a vontade para opinar e sugerir e dificulta seu aprendizado. ${ }^{19}$

Sobre a segunda subcategoria, sentido das orientações após a cirurgia, os pacientes ressaltaram a relação do conhecimento do processo cirúrgico com os sentimentos de tranquilidade, redução da ansiedade e preocupação no pós- operatório. Isso é observado nas seguintes falas, obtidas quando questionados sobre os benefícios percebidos no pós-operatório:

Sim, eu fiquei mais tranquilo porque sabia o que fiz[... ]é muito bom o paciente saber o que ele tem e o que vai ser feito[...] (F7)

Sim. Redução da preocupação e ansiedade (F6)

A preparação psicológica é vista como benéfica se baseada nas necessidades individuais do paciente, pois, ao sentir-se esclarecido em suas dúvidas, diminui-lhe os temores, o que previne possíveis complicações no pós-operatório. Assim, evidencia-se a relação estabelecida entre o conhecimento, o medo e a segurança no pós-operatório. 0 sentimento de medo emerge da cirurgia, dar dor, da anestesia, de não acordar da anestesia, da solidão, dos aparelhos e equipamentos, do resultado da operação e da morte. ${ }^{17}$ Logo, 0 conhecimento do que será feito proporciona segurança e redução do medo.

Além disso, em um estudo ${ }^{17}$ de mesma metodologia com pacientes submetidos à cirurgia cardíaca, os entrevistados relataram a redução da ansiedade e aumento da segurança, pois sabiam o que Ihes seria feito.

$\mathrm{Na}$ 4a Categoria, Sentimentos vivenciados pelo paciente em relação à alta hospitalar, quando os pacientes foram questionados sobre o retorno para seu domicilio, as respostas relacionaram-se com a ansiedade para retornar aos hábitos de vida, como expresso na fala abaixo: 


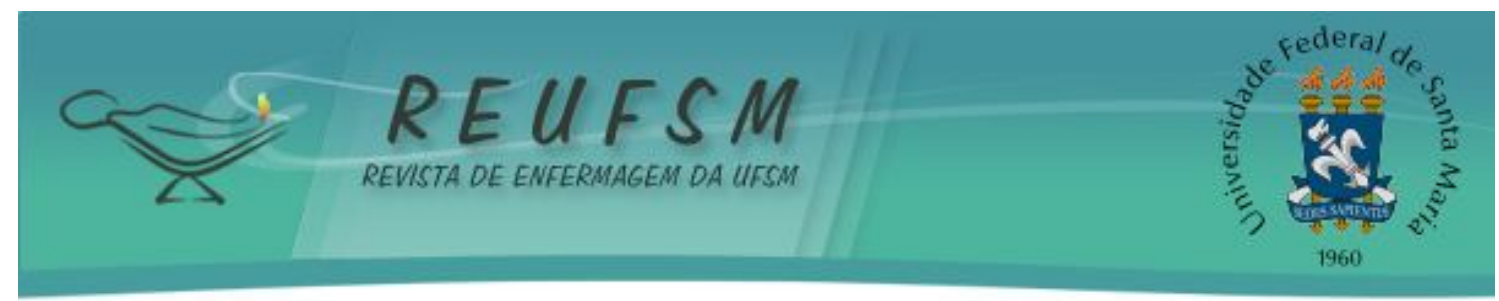

Ansioso, hospital é um lugar horrível, acho que ninguém gosta de ficar isolado aqui, né? (F4)

A hospitalização torna-se desagradável para o indivíduo uma vez que ela causa mudanças nos seus hábitos de vida, bem como o distanciamento de familiares, amigos e de objetos pessoais. ${ }^{20}$ Assim, o hospital é normalmente caracterizado como um ambiente estranho e hostil ao doente e à sua família, que se submetem às rotinas e normas da instituição. A internação também representa mudança brusca do espaço físico, perda da privacidade e da autonomia (capacidade de escolha e decisão), ociosidade e experimentação de reações negativas como ansiedade, depressão, estresse, fobias, medos, angústias e baixa auto-estima. ${ }^{19}$ Assim, o tempo de internação é um aspecto importante.

Embora o paciente já se encontre em pós-operatório tardio, o seu retorno para o domicílio, na maioria dos casos, demanda cuidados importantes com os curativos, a ferida operatória, a postura, atividades físicas e nutrição. No entanto, não se observou referência a esses cuidados domiciliares, o que pode relacionar-se a ansiedade como sentimento de retorno ao domicílio.

Dessa forma, o procedimento cirúrgico é um obstáculo a ser enfrentado pelo paciente. Na sua percepção, após o procedimento, ele estará em condições melhores de saúde, como descrito na fala:

Sinto-me bem para voltar para casa, o pior já passou. (F1)

Por outro lado, o paciente parece não saber que irá precisar de cuidados no domicílio pelo fato de isso não ter sido explicado nas orientações pós-operatórias. Como esse estudo abordou somente as orientações pré- operatórias realizadas na instituição, percebeu-se uma lacuna no que se refere às orientações após a cirurgia. Essas devem ser realizadas quando a alta hospitalar estiver planejada para que o pós-operatório tardio seja mais efetivo e com menos riscos.

Contudo, outro estudo ${ }^{19}$ aponta que os pacientes ainda permanecem com muitas dúvidas e preocupações com relação à dor, prognóstico, morte, família e emprego após o ato cirúrgico. Além disso, as orientações influenciam nas atitudes e comportamentos dos pacientes em relação ao seu autocuidado no pós-operatório e permite que ele se sinta incluído no seu processo de recuperação.

\section{CONCLUSÃO}

A realização desse estudo revelou que a maioria dos pacientes estudados recebeu orientações adequadas sobre suas cirurgias e sentiu-se mais tranquilo e seguro em relação ao procedimento cirúrgico.

Embora as orientações tenham sido realizadas com efeitos positivos no pósoperatório, percebeu-se a necessidade de os profissionais realizarem orientações que se adaptem às necessidades de cada paciente sem definir o grau de importância das informações.

A pesquisa permite inferir que os enfermeiros estão sensibilizados com a diversidade de seus pacientes. Entretanto, percebeu-se a necessidade de os profissionais realizarem orientações que se adaptem às necessidades de cada paciente sem definir 0 grau de importância das informações.

A ansiedade destacou-se como sentimento para alta hospitalar e os cuidados domiciliares foram pouco relatados pelo paciente. Isso sugere uma lacuna nas orientações para a alta hospitalar que não englobaram informações sobre esses cuidados. 


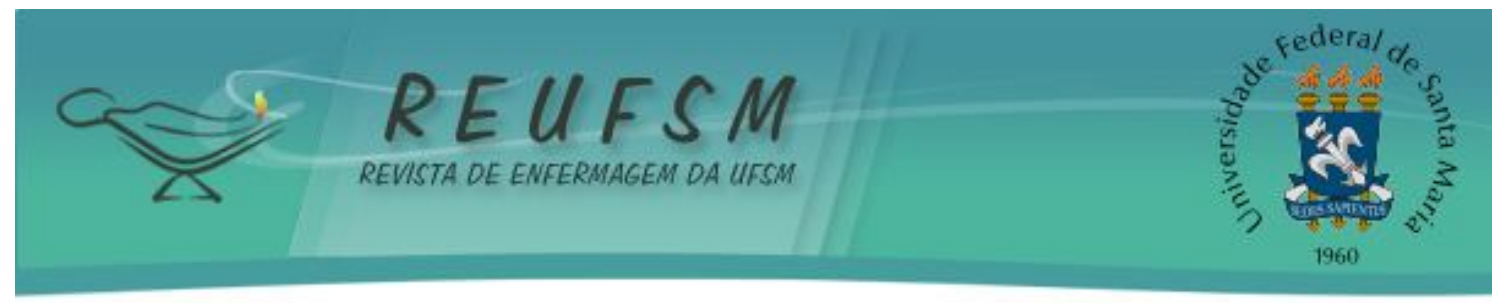

Dessa forma, a segurança e a tranquilidade foram os sentimentos revelados pelos pacientes com relação à orientação recebida. Eles percebem o profissional que realizou as orientações pré-operatórias como atencioso, simpático e bondoso. O sentido das orientações antes da cirurgia foi a obtenção de informações sobre o procedimento cirúrgico. Após o ato cirúrgico, os pacientes ressaltaram a relação do conhecimento dessas informações com os sentimentos de tranqüilidade, redução da ansiedade e preocupação no pós- operatório. Os sentimentos vivenciados pelo paciente em relação à alta hospitalar foram de ansiedade para retornar ao domicílio e aos seus hábitos de vida.

A partir desses resultados, sugere-se a realização de estudos que explorem as orientações com enfoque para a alta hospitalar e cuidados domiciliares, visto a lacuna encontrada nessa fase do perioperatório. Além disso, com essa pesquisa, pretende-se estimular os enfermeiros para a necessidade de reconstrução de novas práticas no cuidado aos pacientes cirúrgicos, com garantia do direito de atendimento conforme as suas necessidades e de uma assistência de enfermagem com qualidade.

\section{REFERÊNCIAS}

1. Sena RR, Silva KL, Gonçalves AM, Duarte ED, Coelho S. O cuidado no trabalho em saúde: implicações para a formação de enfermeiros. Interface (Botucatu). 2008; 12(24): 23-34.

2. Pereira SE. Contribuições para um planejamento educacional em ciências da saúde com estratégias inovadoras de ensino-aprendizagem. Com ciênc saúde. 2007; 18(1): 33-44.

3. Assis M. Promoção da saúde e envelhecimento: avaliação de uma experiência no ambulatório do núcleo de atenção ao idoso da UnATi/UERJ. Rio de Janeiro (RJ): [s.n.], 2004. 220 p. Tese (doutorado) - Escola Nacional de Saúde Pública, Fundação Oswaldo Cruz Fiocruz; 2004.

4. Lopes EM, Anjos SJ SB, Pinheiro AKB. Tendência das ações de educação em saúde realizadas por enfermeiros no brasil. Rev enferm UERJ . 2009; 17(2):273-7.

5. Cervera DPP, Parreira BDM, Goulart BF. Educação em saúde: percepção dos enfermeiros da atenção básica em Uberaba (MG). Ciênc saúde coletiva. 2011;16Supl 1:1547-54.

6. Grazinelli MF, Reis DC, Marques RC. Educação em saúde: teoria, métodos e imaginação. Belo Horizonte: UFMG; 2006. p. 43-51.

7. Christóforo BEB, Carvalho DS. Cuidados de enfermagem realizados ao paciente cirúrgico no período pré-operatório. Rev Esc Enferm USP. 2009; 43(1):14-22.

8. Paula AAD, Carvalho EC. Ensino sobre perioperatório a pacientes: estudo comparativo de recursos audiovisual (vídeo) e oral. Rev latinoam enferm. 1997; 5(3): 35-42.

9. Lima FB, Silva J LL, Gentile AC. A relevância da comunicação terapêutica na amenização do estresse de clientes em pré-operatório: cuidando através de orientações. Rev Informese em promoção da saúde. 2007; 3(2): 17-8.

10. Setor Estatístico do Hospital Universitário de Santa Maria. Cirurgias de Esofagectomia e Gastrectomia: Registro de Cirurgias do HUSM. Santa Maria(RS): HUSM; 2010.

11. Instituto Nacional de Câncer; Ministério da Saúde. Câncer no Brasil: dados dos registros de base populacional. Rio de J aneiro: INCA; 2003.

12. Bardin L. Análise de conteúdo. Lisboa: Edições 70. 1979. 


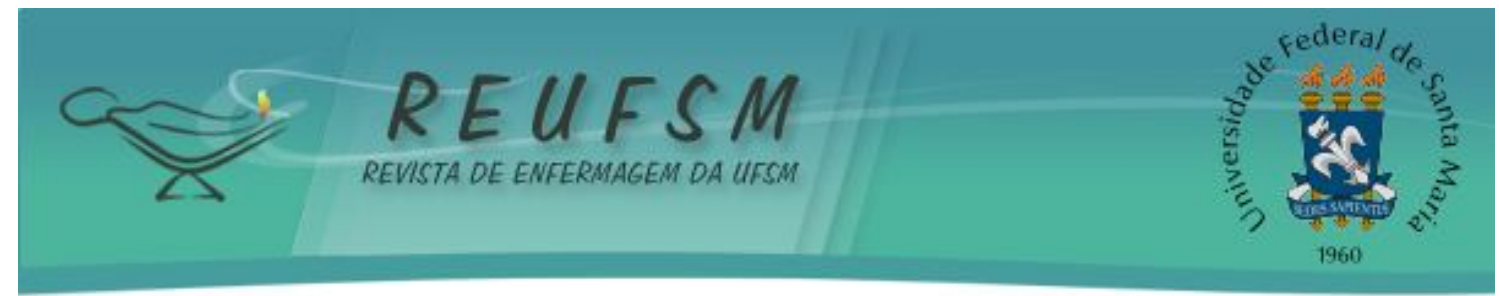

13. Ministério da Saúde (BR). Conselho Nacional de Saúde. Diretrizes e normas regulamentadoras de pesquisa envolvendo seres humanos. Resolução n. 196, de 10 de outubro de 1996. Brasília; 1996.

14. Merhy EE. Saúde: a cartografia do trabalho vivo. 2ª̣ed. São Paulo: Hucitec; 2002.

15. Perrando MS, Beuter M, Brondani CM, Roso CC, Santos TM, Predebon GR. O preparo pré-operatório na ótica do paciente cirúrgico. R Enferm UFSM. 2011; 1(1): 61-70.

16. Biazin DT, Coldibelli LMF, Ribeiro RP, Recanello J, Simon MCF, Silva MC et. al. Projeto de extensão: visita pré e pós-operatória de cirurgia cardíaca. Terra e Cultura. 2002; (35): 133-142, 2002.

17. Nascimento DD, Correia DMS. A percepção do cliente hospitalizado na interação interpessoal com os profissionais de saúde. Rev enferm UFPE on line [periódico na internet]. 2011[acesso em 15 de Agosto de 2010];5(2):238-47. Disponível em: http:/ / www. ufpe. br/ revistaenfermagem/ index. php/ revista/ article/ view/ 1528

18. Lima FB, Silva JLL, Gentile AC. A relevância da comunicação terapêutica na amenização do estresse de clientes em pré-operatório: cuidando através de orientações. Rev Informe-se em promoção da saúde. 2007; 3(2): 17-8.

19. Kruse MHL, Almeida MA, Keretzky KB, Rodrigues E, Silva FP, Schenini FS, Garcia VM. Orientação pré-operatória da enfermeira: lembranças de pacientes. Rev eletrônica enferm [periódico na internet]. 2009[acesso em 10 de Setembro de 2010]; 11(3): 494-500. Disponível em: http:/ / www.fen.ufg.br/ revista/ v11/ n3/ pdf/ v11n3a05. pdf

20. Marin MJ S. Preparando o idoso para a alta hospitalar. Ribeirão Preto: [s.n.], 1999. 190 p. Dissertação (Mestrado) - Universidade de São Paulo. Escola de Enfermagem de Ribeirão Preto, 1999.

Data de recebimento: $07 / 07 / 2011$

Data de aceite: 13/10/2011

Contato com autor responsável: Rodrigo Marques da Silva.

Endereço Postal: Rua Conde de Porto Alegre, 1240. Apto 203. Bairro Centro. Santa Maria, RS. CEP: 97015110.

E-mail: marques-sm@hotmail.com 